\title{
WHAT OPTIONS DOES THE SUPINE-STANDING-SUPINE TEST OFFER TO PATIENTS WITH HYPERTENSION? DEMONSTRATIONS OF EXAMPLES.
}

\author{
Jan Galuszka
}

\author{
$I^{s t}$ Department of Internal Medicine, Teaching Hospital and Palacky University, Olomouc, Czech Republic \\ e-mail: Jan.Galuszka@fnol.cz
}

Received: September 2, 2007; Accepted: October 8, 2007

Keywords: Autonomic nervous system/Hypertension/Heart rate variability/Supine-standing-supine test/Spectral analysis/ Age-standardised parameters

Aims: The purpose of the study was to demonstrate autonomic nervous system (ANS) changes associated with treatment in hypertensive patients and utilization of these measurements in practice.

Methods: Hypertensive patients were examined before the start of treatment and after blood pressure compensation. The telemetric system VarCor PF 5 was used for non-invasive heart rate registration and automatic evaluation of heart rate variability (HRV) parameters. The supine-standing-supine test with ortho-clinostatic loading in standard conditions was used for HRV evaluation.

Results: The influence of antihypertensive therapy on ANS was demonstrated in two cases. The first was a case of a responder to therapy and the second case was example of a non-responder to monotherapy regarding more therapeutic steps for blood pressure control. Different modes of results presentation are demonstrated: 3D graph, numeric form of standard parameters of HRV, computer interpretation of results by means of complex parameters and indices in numeric, graphic and verbal form with functional age calculation, cross graph of vagal activity index versus sympatho-vagal balance index was used for repeated measurements and follow-up. The increased spectral performance with augmented vagal activity and slope down of sympatho-vagal ratio are evident after blood pressure control achievement. Improvement of functional age parameter associated with blood pressure compensation is visible using a complex age-dependent parameters interpretation.

Conclusions: HRV evaluation in hypertensive patients provides new parameters for patient's examination. These parameters are influenced by both the illness itself and by pharmacotherapy. Such an approach offers more complex information about patient.

\section{INTRODUCTION}

Blood pressure (BP) is the result of peripheral vascular resistance, central venous pressure and cardiac output interactions. It is self-evident that heart rate is an important parameter of actual circulatory compensation as cardiac output is dependent on heart beat frequency. Blood pressure regulation in hypertensive patients is still inadequate despite increasing knowledge of the aethiopathogenesis of this condition and the wide range of pharmacotherapeutic modalities available. Blood pressure is continuously under the control of multiple regulatory processes including the ANS as a major regulatory and integrative system ${ }^{1}$. Heart rate variability (HRV) measurement is a well-defined noninvasive method of evaluating the ANS $\left(\right.$ ref. $\left.^{2}\right)$. Spectral analysis (SA) of HRV enables us to distinguish three main spectral components from short-term recordings: a high frequency (HF) component $(0.15-0.40 \mathrm{~Hz})$, a low frequency (LF) component $(0.05-0.15 \mathrm{~Hz})$ and a very low frequency (VLF) component $(\leq 0.04 \mathrm{~Hz})\left(\right.$ ref. $\left.^{2,3}\right)$. The supine-standing-supine test is a mode of evaluating the short-term HRV records using standardised orthostatic loading for facilitation of sympatho-vagal balance. Detailed analysis of large number of HRV parameters can be simplified using age-standardised parameters in the spectral analysis of HRV and by the method of complex indices assessment ${ }^{4}$.

\section{METHODS AND CLINICAL APPLICATION}

The activity of the ANS is dynamic variable representing the reaction of ANS to currently operating external and internal stimuli in interaction with responses to stimuli acted in past ${ }^{5}$. Therefore the supine-standing-supine test was used for HRV evaluation. This test consists in assessment of autonomic reactions based on short-term record of heart rate in defined conditions. Examined patient stayed 5 minutes in every position for 300 RR intervals recording, value of "window" parameter was 256 (ref. ${ }^{6-8}$ ). Record of RR intervals and HRV analysis were provided by using of VarCor PF5 microcomputer system for telemetric assessment of short term heart rate variability in time and frequency domain with application of fast Fourier transformation and partially modified algorithm CGSA (coarse-graining spectral analysis) ${ }^{9}$. Measured parameters with similar course and age dependency were processed by method of complex indicators with calculation so called functional age by method of Stejskal and Šlachta ${ }^{5,10,11}$. 
Four types of results were assessed:

1) Three dimensional (3D) graph of HRV.

2) Results in numerical form. Parameters: RR (ms), Power VLF $\left(\mathrm{ms}^{2}\right)$, Power LF $\left(\mathrm{ms}^{2}\right)$, Power HF $\left(\mathrm{ms}^{2}\right)$, Power spectral density - PSD VLF $\left(\mathrm{ms}^{2} / \mathrm{Hz}\right)$, PSD LF $\left(\mathrm{ms}^{2} / \mathrm{Hz}\right)$, PSD HF $\left(\mathrm{ms}^{2} / \mathrm{Hz}\right)$, Frequency VLF $(\mathrm{Hz})$, Frequency LF (Hz), Frequency $\mathrm{HF}(\mathrm{Hz})$, Coeficient of variance - CCV VLF, CCV LF, CCV HF, Relative VLF (\%), Relative LF (\%), Relative HF (\%), VLF/HF, $\mathrm{LF} / \mathrm{HF}, \mathrm{VLF} / \mathrm{LF}, \mathrm{MSSD}\left(\mathrm{ms}^{2}\right)$, Total power $\left(\mathrm{ms}^{2}\right)$.

3) Results processed by mean of age-standardised parameters with calculation of functional age with computer interpretation of ANS outcomes in numeric, graphic and verbal forms.

4) Points of intersection of complex indices: vagal activity index versus sympatho-vagal balance index in cross graph for longitudinal follow-up.

The above mentioned testing applied in ten previously healthy persons (without any pharmacotherapy) with new diagnosis of hypertension indicated for pharmacotherapy. Thus the only variable was new medicament therapy. All patients were in sinus rhythm. All patients were examined in the morning time fasting, with omission of tabaco, coffee and alcohol. The first examinations were provided before start of pharmacotherapy and control examinations were performed from one to three weeks later when blood pressure control was provided.

\section{RESULTS}

There is possible to find two different situations in the group of hypertensive patients. On the one hand we can find good response to started therapy and $\mathrm{BP}$ value in control examination in normal range. On the other hand control BP measurement reveals persistent hypertension despite of therapy and next therapeutic step is necessary. Both cases are demonstrated with their findings in supine-standing-supine test. The first case presents woman with good response to betablocker administration: Figures 1-3. The opposite situation demonstrates man without BP improvement after betablocking agent administration and there were three therapeutic steps necessary to achieve target BP level: Figures 4-6, Table 1.

Fig. 1. 3-D graph of SA HRV during supine-standing-supine test in woman 27 years. Legend: $\mathrm{x}$ axis frequency (Hz), $\mathrm{y}$ axis power spectral density $(\mathrm{ms} / \mathrm{Hz}), \mathrm{z}$ axis time course of test T1 supine, T2 standing, T3 supine (each 300 seconds). Enhanced total spectral power and especially HF component are markedly visible.

A. Before treatment BP $140 / 90 \mathrm{mmHg}$ (therapy indication according to BP Holter monitoring).

B. Control examination with blood pressure compensation on Bisoprolol 5mg daily BP 120/70 $\mathrm{mmHg}$.

A

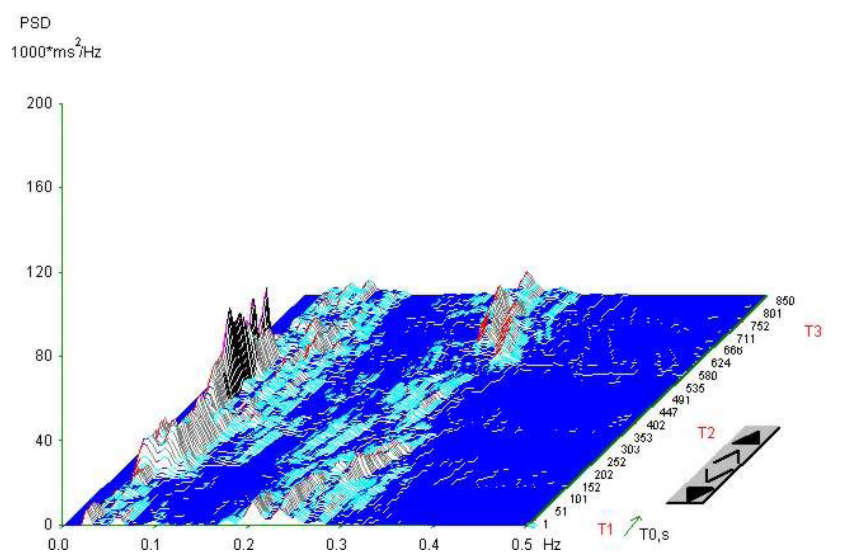

B

PSD

$1000^{*} \mathrm{~ms}^{2} \cdot \mathrm{Hz}$

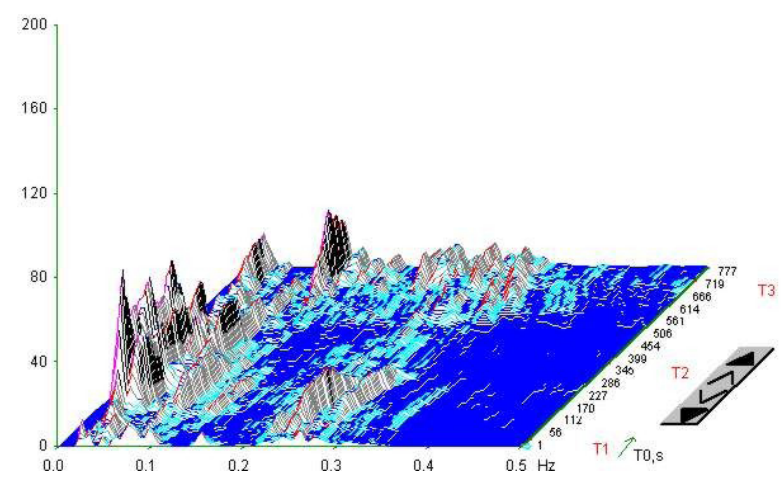


Fig. 2. Numerical form of SA HRV. Hypertensive woman 27 years. Markable rising of all spectral component especially in supine position where are approximately twofold values.

A1. Before treatment - standing position.

A2. Before treatment - final supine position.

B1. Control examination with blood pressure compensation on Bisoprolol $5 \mathrm{mg}$ daily - standing position.

B2. Control examination with blood pressure compensation on Bisoprolol $5 \mathrm{mg}$ daily final - supine position.

A1

\begin{tabular}{|c|c|c|c|c|c|}
\hline \multicolumn{4}{|c|}{ Record: 0001 filtered (8.11.2005 8:17:32) } & \multicolumn{2}{|c|}{ Hardware: VarCor PF6 } \\
\hline \multicolumn{6}{|c|}{$\begin{array}{l}\text { Analysis type: VLF, window: } 256 \quad \text { Analysis date: } 30.4 .200 \\
\text { Frequency bands borders [Hz]: VLF: } 0,020, \text { LF: } 0,050, \text { HF: } 0,150\end{array}$} \\
\hline \multicolumn{4}{|c|}{ Frequency axis $[\mathrm{Hz}]: 0,500$} & \multicolumn{2}{|c|}{ Interval: } \\
\hline & Average & St. dev. & St. deu. \% & & \\
\hline Power VLF & 165,33 & 10,55 & 6,38 & CCV VLF & 1,7428 \\
\hline Power LF & 235,48 & 21,91 & 9,30 & CCV LF & 2,0799 \\
\hline Power HF & 261,61 & 3,51 & 1,34 & CCV HF & 2,1923 \\
\hline PSD VLF & 10505,81 & 1305,19 & 12,42 & Rel. VLF & 24,96 \\
\hline PSD LF & 6016,27 & 371,00 & 6,17 & Rel. LF & 35,55 \\
\hline PSD HF & 4632,47 & 144,48 & 3,12 & Rel. HF & 39,49 \\
\hline Freq. VLF & 36,09 & 1,98 & 5,49 & & \\
\hline Freq. LF & 78,65 & 6,59 & 8,38 & MIM50 & 4 \\
\hline Freq. HF & 258,81 & 1,33 & 0,51 & pkin50 & 1,33 \\
\hline VLFAF & 0,6317 & 0,0352 & 5,57 & MSSD & 411,52 \\
\hline LFAF & 0,9004 & 0,0864 & 9,59 & Total power & 662,42 \\
\hline VLF/LF & 0,7101 & 0,0946 & 13,32 & & \\
\hline RR & 0,7378 & 0,0358 & 4,85 & Breath freq. & $2 ? ?$ \\
\hline
\end{tabular}

B1

\begin{tabular}{|c|c|c|c|c|c|}
\hline \multicolumn{4}{|c|}{ Record: 0001 filtered (28.11.2005 8:15:32) } & \multicolumn{2}{|c|}{ Hardware: VarCor PF6 } \\
\hline \multirow{2}{*}{\multicolumn{6}{|c|}{$\begin{array}{l}\text { Analysis type: VLF, window: } 256 \\
\text { Frequency bands borders }[\mathrm{Hz}] \text {. VLF: } 0,020, \text { LF: } 0,050, \mathrm{HF}: 0,150\end{array}$}} \\
\hline & & & & & \\
\hline \multicolumn{4}{|c|}{ Frequency axis $[\mathrm{Hz}]: 0,500$} & \multicolumn{2}{|c|}{ Interval: 2} \\
\hline & Average & St. dev. & St. deu. \% & & \\
\hline Power VLF & 463,62 & 28,20 & 6,08 & CCV VLF & 2,4134 \\
\hline Power LF & 647,53 & 20,34 & 3,14 & CCV LF & 2,8521 \\
\hline Power HF & 247,23 & 5,51 & 2,23 & CCV HF & 1,7624 \\
\hline PSD VLF & 36111,85 & 5682,17 & 15,73 & Rel. VLF & 34,13 \\
\hline PSD LF & 23889,49 & 1063,06 & 4,45 & Rel. LF & 47,67 \\
\hline PSD HF & 3696,38 & 548,15 & 14,83 & Rel. HF & 18,20 \\
\hline Freq. VLF & 28,71 & 3,41 & 11,89 & & \\
\hline Freq. LF & 73,01 & 8,64 & 11,84 & HN50 & 11 \\
\hline Freq. HF & 240,64 & 8,25 & 3,43 & pHIN50 & 3,67 \\
\hline VLFHF & 1,8766 & 0,1278 & 6,81 & MSSD & 670,53 \\
\hline LFAHF & 2,6214 & 0,1239 & 4,73 & Total power & 1358,39 \\
\hline VLF/LF & 0,7155 & 0,0264 & 3,69 & & \\
\hline RR & 0,8922 & 0,0549 & 6,15 & Breath freq. & ??? \\
\hline
\end{tabular}

A2

\begin{tabular}{|c|c|c|c|c|c|}
\hline \multicolumn{4}{|c|}{ Record: 0001 filtered (8.11.2005 8:17:32) } & \multicolumn{2}{|c|}{ Hardware: VarCor PF6 } \\
\hline \multirow{2}{*}{\multicolumn{6}{|c|}{$\begin{array}{l}\text { Analysis type: VLF, window: } 256 \quad \text { Analysis date: } 30.4 .200713: 46: 34 \\
\text { Frequency bands borders [Hz]. VLF: } 0,020, \text { LF: } 0,050, \text { HF: } 0,150 \\
\text { Frequency axis [Hz] } 0,500\end{array}$}} \\
\hline & & & & & \\
\hline & Average & St. dev. & St. deu. \% & & \\
\hline Power VLF & 68,67 & 14,08 & 20,50 & CCV VLF & 0,9904 \\
\hline Power LF & 159,04 & 15,58 & 9,80 & CCVLF & 1,5073 \\
\hline Power HF & 432,69 & 10,91 & 2,52 & CCV HF & 2,4861 \\
\hline PSD VLF & 4002,70 & 1094,25 & 27,34 & Rel. VLF & 10,40 \\
\hline PSD LF & 4907,56 & 259,82 & 5,29 & Rel. LF & 24,08 \\
\hline PSD HF & 14739,46 & 1297,96 & 8,81 & Rel. HF & 65,52 \\
\hline Freq. VLF & 40,35 & 2,16 & 5,36 & & \\
\hline Freq. LF & 85,89 & 3,56 & 4,15 & HM50 & 25 \\
\hline Freq. HF & 237,46 & 1,11 & 0,47 & phin50 & 8,33 \\
\hline VLFAHF & 0,1596 & 0,0370 & 23,21 & MSSD & 904,37 \\
\hline LFAHF & 0,3684 & 0,0442 & 12,01 & Total power & 660,40 \\
\hline VLF/LF & 0,4290 & 0,0555 & 12,93 & & \\
\hline RR & 0,8367 & 0,0388 & 4,64 & Breath freq. & $2 ? ?$ \\
\hline
\end{tabular}

Units: Power [ms2], PSD [ms2 $\mathrm{Hz}]$, Freq $[\mathrm{mHz}], \mathrm{R}-\mathrm{R}[\mathrm{s}]$, Ereath freq. [breathsinin]

\section{B2}

\begin{tabular}{|c|c|c|c|c|c|}
\hline \multicolumn{4}{|c|}{ Record: 0001 filtered (28.11.2005 8:15:32) } & \multicolumn{2}{|c|}{ Hardware: VarCor PF6 } \\
\hline \multicolumn{6}{|c|}{$\begin{array}{l}\text { Analysis type: VLF, window: } 256 \\
\text { Frequency bands borders [Hz] VLF: } 0,020, \text { LF: } 0,050 \text {, HF: } 0,150\end{array}$} \\
\hline \multicolumn{4}{|c|}{ Frequency axis $[\mathrm{Hz}]: 0,500$} & \multicolumn{2}{|c|}{ Interval: $3=$} \\
\hline & Average & St. dev. & St. deu. \% & & \\
\hline Power VLF & 168,49 & 15,75 & 9,35 & CCV VLF & 1,3223 \\
\hline Power LF & 439,67 & 44,69 & 10,16 & CCV LF & 2,1361 \\
\hline Power HF & 1138,57 & 71,49 & 6,28 & CCV HF & 3,4374 \\
\hline PSD VLF & 16895,18 & 415,12 & 2,46 & Rel. VLF & 9,65 \\
\hline PSD LF & 29068,70 & 1796,43 & 6,18 & Rel. LF & 25,17 \\
\hline PSD HF & 13443,01 & 417,42 & 3,11 & Rel. HF & 65,18 \\
\hline Freq. VLF & 24,03 & 0,94 & 3,89 & & \\
\hline Freq. LF & 107,14 & 0,00 & 0,00 & HN50 & 78 \\
\hline Freq. HF & 230,16 & 16,67 & 7,24 & pNW50 & 26,00 \\
\hline VLFAHF & 0,1478 & 0,0060 & 4,08 & MSSD & 3295,70 \\
\hline LF/HF & 0,3881 & 0,0516 & 13,30 & Total power & 1746,73 \\
\hline VLF/LF & 0,3886 & 0,0633 & 16,28 & & \\
\hline RR & 0,9816 & 0,0557 & 5,68 & Breath freq. & ??? \\
\hline
\end{tabular}

Units: Power [ms2], PSD [ms2 $\mathrm{Hz}]$, Freq [mHz], R-R [s], Ereath freq. [breathsimin] 
Fig. 3. Computer interpretation of ANS evaluation by method of complex parameters and indices in numeric, graphic and verbal form with so called functional age calculation. Woman 27 years. After BP compensation so called functional age is appropriate to calendar age.

A. Before treatment.

B. Woman 27 years BP compensation on Bisoprolol $5 \mathrm{mg}$ daily.

$\mathbf{A}$

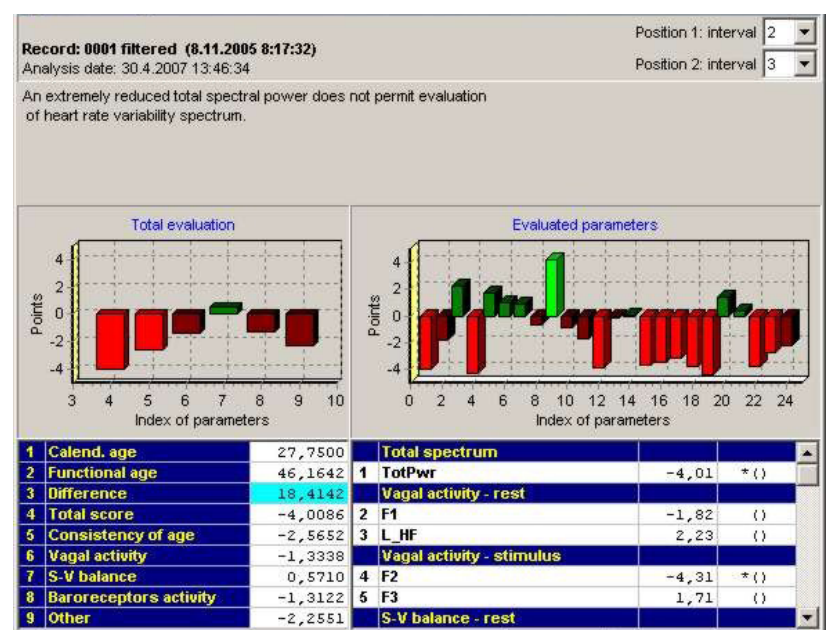

B

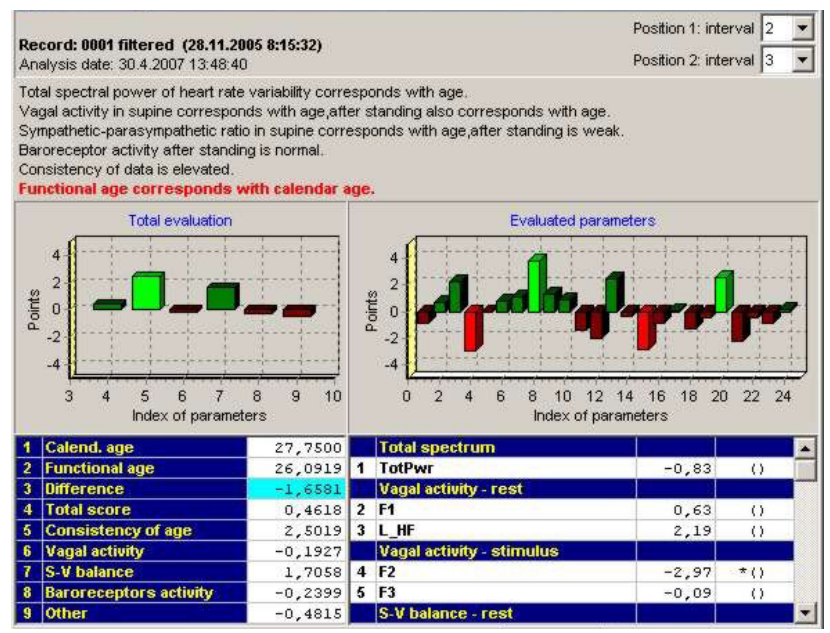

Fig. 4. Graphic form of SA HRV during supine-standing-supine test. Man 39 years "non responder" to monotherapy. Therapeutic success is associated with increased spectral activity at final examination - D.

A. Examination before treatment BP $180 / 110 \mathrm{mmHg}$.

B. Betaxolol $20 \mathrm{mg}$ with BP $160 / 110 \mathrm{mmHg}$.

C. Betaxolol $20 \mathrm{mg}$ and Ramipril $5 \mathrm{mg}$ BP 150/100 $\mathrm{mmHg}$.

D. Final result with BP 140/83 mmHg under control on combination of Betaxolol $20 \mathrm{mg}$ and Ramipril 10mg daily.

A

${ }_{1000^{*} \mathrm{~ms}^{2} \mathrm{HHz}}^{\mathrm{PSD}}$

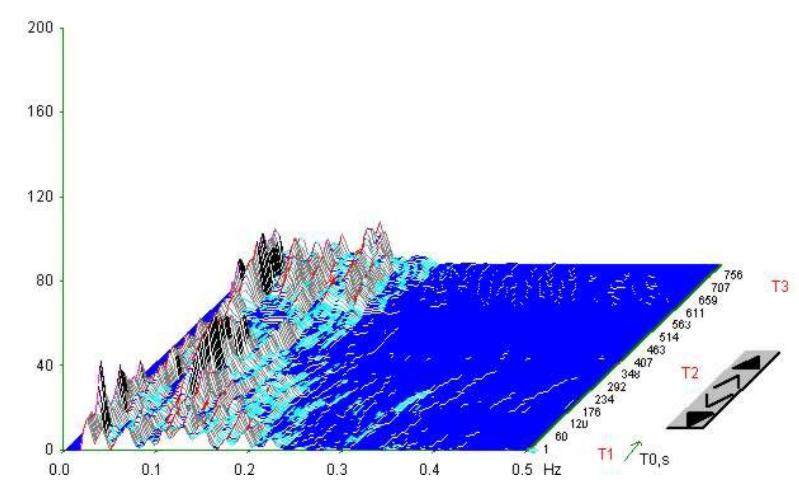

\section{C}

PSD
$1000^{*} \mathrm{~ms}^{2} \mathrm{HHz}$

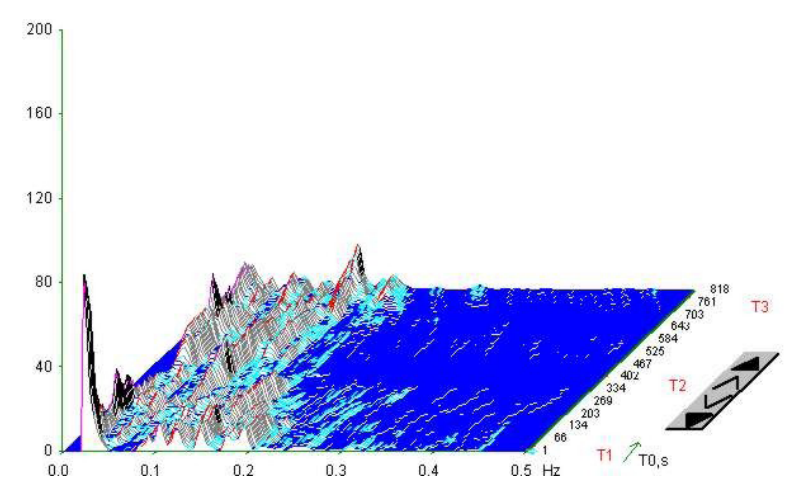

B

PSD

$1000^{*} \mathrm{~ms}^{2} \mathrm{iHz}$

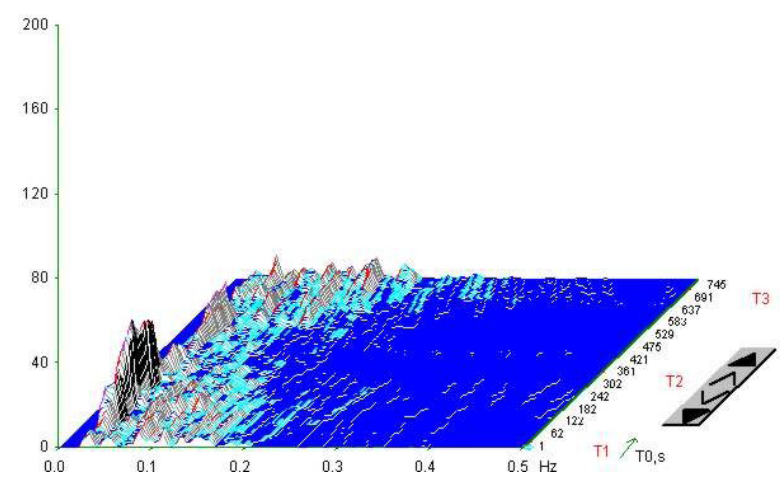

D

PSD
$1000^{*} \mathrm{~ms}^{2} \mathrm{iHz}$

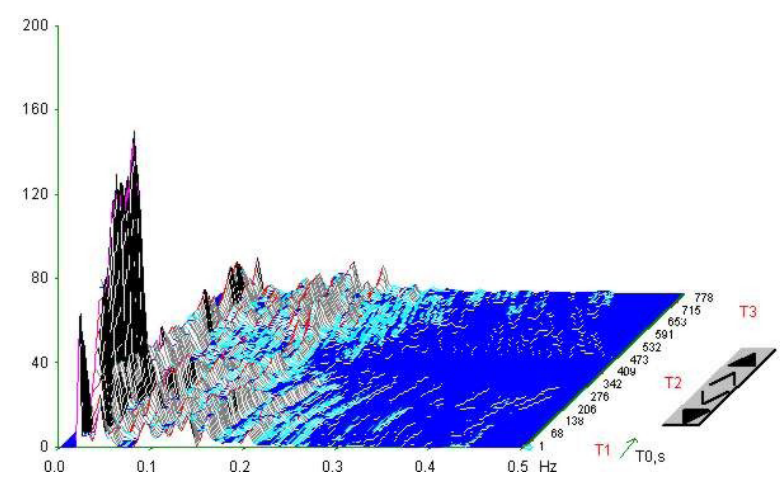


Table 1. Numerical form of SA HRV. Man 39 years “non responder" to monotherapy. The results demonstrate high LF/HF ratio (sympathovagal balance) and weak total spectral performance before treatment and gradual improvement of spectral performance and especially HF component (CCV HF) during therapy.

\begin{tabular}{|c|c|c|c|c|}
\hline \multicolumn{5}{|c|}{$\begin{array}{l}\text { Changes of average values of SA HRV results in standing position during supine-standing-supine test } \\
\text { in "non-responder" to therapy. Units: Power }\left(\mathrm{ms}^{2}\right) \text {, PSD }\left(\mathrm{ms}^{2} / \mathrm{Hz}\right) \text {, Frequency }(\mathrm{Hz}), \mathrm{MSSD}\left(\mathrm{ms}^{2}\right) \text {. }\end{array}$} \\
\hline & Before treatment & Betaxolol $20 \mathrm{mg}$ & $\begin{array}{l}\text { Betaxolol } 20 \mathrm{mg} \\
\text { Ramipril } 5 \mathrm{mg}\end{array}$ & $\begin{array}{l}\text { Betaxolol } 20 \mathrm{mg} \\
\text { Ramipril } 10 \mathrm{mg}\end{array}$ \\
\hline Power VLF & 94.68 & 184.71 & 97.62 & 211.02 \\
\hline Power LF & 441.39 & 125.41 & 285.71 & 280.05 \\
\hline Power HF & 78.55 & 43.03 & 57.10 & 125.81 \\
\hline PSD VLF & 7132.61 & 12109.15 & 5781.95 & 18066.58 \\
\hline PSD LF & 14630.74 & 5148.76 & 13780.51 & 6387.71 \\
\hline PSD HF & 1927.80 & 722.98 & 1385.24 & 6982.08 \\
\hline Freq. VLF & 24.21 & 38.48 & 39.94 & 24.41 \\
\hline Freq. LF & 103.49 & 53.43 & 97.75 & 109.24 \\
\hline Freq. HF & 163.68 & 178.88 & 172.0 & 154.18 \\
\hline $\mathrm{VLF} / \mathrm{HF}$ & 1.2071 & 4.37 & 1.7955 & 1.6891 \\
\hline $\mathrm{LF} / \mathrm{HF}$ & 5.6109 & 2.9329 & 5.1252 & 2.2485 \\
\hline VLF/LF & 0.2160 & 1.4901 & 0.3380 & 0.7540 \\
\hline CCV VLF & 1.1472 & 1.4785 & 0.9815 & 1.4256 \\
\hline CCV LF & 2.4771 & 1.2183 & 1.679 & 1.6423 \\
\hline $\mathrm{CCV} \mathrm{HF}$ & 1.0450 & 0.7136 & 0.7506 & 1.1008 \\
\hline Rel. VLF & 15.40 & 52.3 & 22.17 & 34.21 \\
\hline Rel. LF & 71.62 & 35.51 & 64.87 & 45.40 \\
\hline Rel. HF & 12.78 & 12.19 & 12.96 & 30.39 \\
\hline Total power & 614.62 & 353.14 & 440.43 & 616.88 \\
\hline MSSD & 352.5 & 359.33 & 346.84 & 549.05 \\
\hline
\end{tabular}

\begin{tabular}{|c|c|c|c|c|}
\hline \multicolumn{5}{|c|}{$\begin{array}{l}\text { Changes of average values of SA HRV results in final supine position during supine-standing-supine test } \\
\text { in "non-responder" to therapy. Units: Power }\left(\mathrm{ms}^{2}\right), \operatorname{PSD}\left(\mathrm{ms}^{2} / \mathrm{Hz}\right) \text {, Frequency }(\mathrm{Hz}), \mathrm{MSSD}\left(\mathrm{ms}^{2}\right) \text {. }\end{array}$} \\
\hline & Before treatment & Betaxolol $20 \mathrm{mg}$ & $\begin{array}{l}\text { Betaxolol } 20 \mathrm{mg} \\
\text { Ramipril } 5 \mathrm{mg}\end{array}$ & $\begin{array}{l}\text { Betaxolol } 20 \mathrm{mg} \\
\text { Ramipril } 10 \mathrm{mg}\end{array}$ \\
\hline Power VLF & 242.51 & 85.1 & 154.96 & 173.18 \\
\hline Power LF & 703.40 & 404.58 & 503.92 & 432.33 \\
\hline Power HF & 215.59 & 312.25 & 283.47 & 397.43 \\
\hline PSD VLF & 13730.89 & 5742.66 & 14201.2 & 15484.24 \\
\hline PSD LF & 15966.1 & 8429.26 & 16507.8 & 13575.32 \\
\hline PSD HF & 7556.72 & 9632.7 & 7898.5 & 6803.03 \\
\hline Freq. VLF & 38.81 & 45.65 & 21.58 & 26.54 \\
\hline Freq. LF & 108.11 & 107.8 & 133.22 & 136.74 \\
\hline Freq. HF & 151.25 & 154.5 & 176.2 & 163.24 \\
\hline $\mathrm{VLF} / \mathrm{HF}$ & 1.135 & 0.2744 & 0.5457 & 0.4379 \\
\hline $\mathrm{LF} / \mathrm{HF}$ & 3.2886 & 1.3024 & 1.7803 & 1.0912 \\
\hline VLF/LF & 0.3457 & 0.2105 & 0.3098 & 0.4006 \\
\hline CCV VLF & 1.6716 & 0.9031 & 1.1511 & 1.1248 \\
\hline CCV LF & 2.8468 & 1.9692 & 2.0759 & 1.771 \\
\hline CCV HF & 1.5761 & 1.73 & 1.5570 & 1.7039 \\
\hline Rel. VLF & 20.88 & 10.61 & 16.44 & 17.27 \\
\hline Rel. LF & 60.56 & 50.45 & 53.47 & 43.11 \\
\hline Rel. HF & 18.56 & 38.94 & 30.08 & 39.63 \\
\hline Total power & 1161.50 & 801.93 & 942.35 & 1002.94 \\
\hline MSSD & 776.81 & 797.54 & 851.19 & 1472.57 \\
\hline
\end{tabular}


Fig. 5. Computer interpretation of ANS evaluation by method of complex parameters and indices in numeric, graphic and verbal form with so called functional age calculation. Man 39 years "non responder" to monotherapy. Changes in complex parameters during therapy with final improvement of functional age parameter are demonstrated.

A. Examination before treatment BP $180 / 110 \mathrm{mmHg}$.

B. Betaxolol $20 \mathrm{mg}$ with BP $160 / 110 \mathrm{mmHg}$.

C. Betaxolol $20 \mathrm{mg}$ and Ramipril $5 \mathrm{mg}$ with BP 150/100 $\mathrm{mmHg}$.

D. Final result with BP 140/83 mmHg under control on combination of Betaxolol $20 \mathrm{mg}$ and Ramipril $10 \mathrm{mg}$ daily.

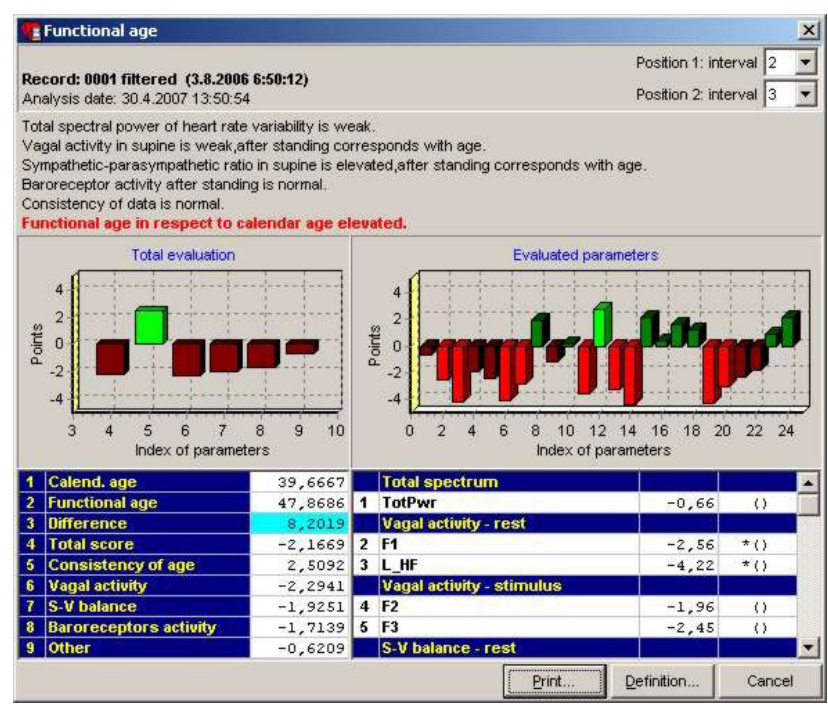

$\mathbf{A}$

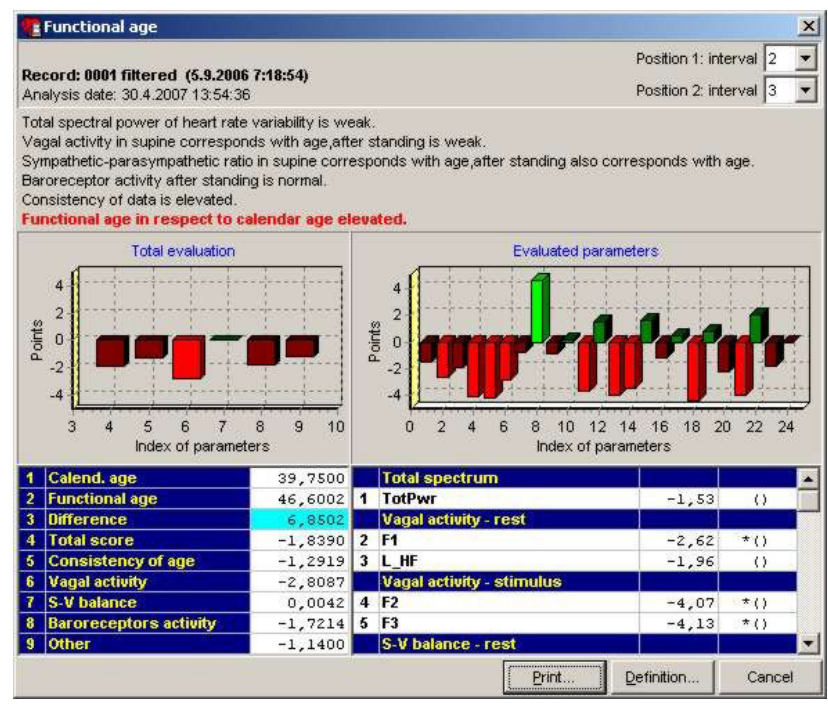

C

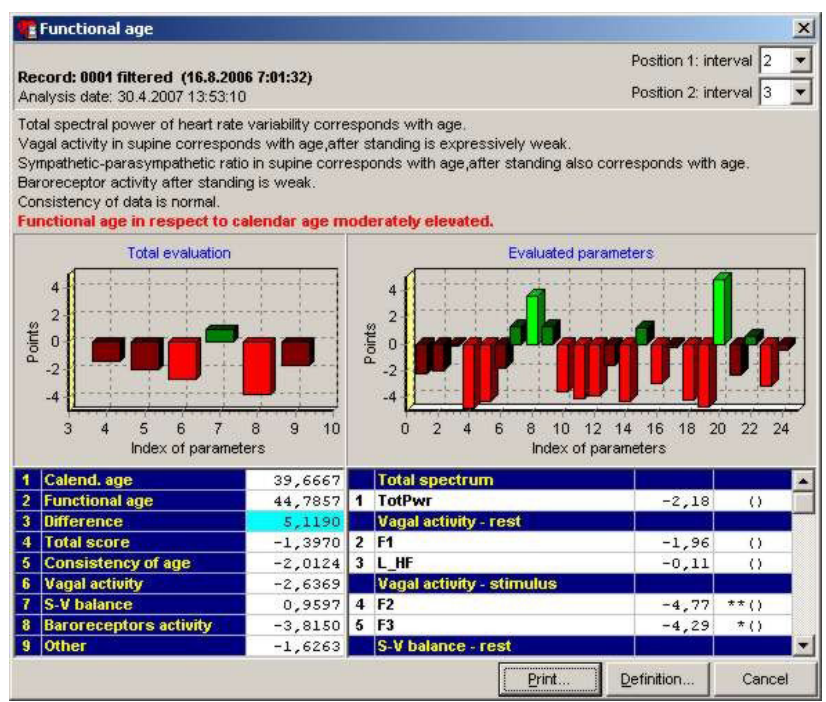

B

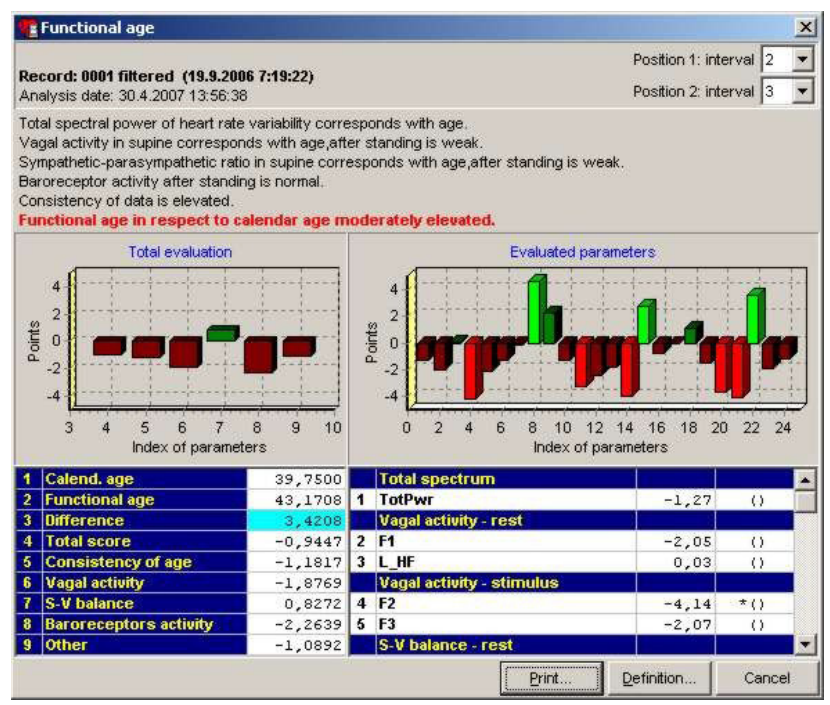

D 
Fig. 6. Longitudinal follow-up of patient with hypertension by assessment of comlex HRV indices expressed in cross graph. Man 39 years "non responder" to monotherapy. Legend: axis $\mathrm{x}$ vagal activity index, axis y sympatho-vagal balance index.

1. Before treatment BP $180 / 110 \mathrm{mmHg}$.

2. Betaxolol $20 \mathrm{mg}$ BP $160 / 110 \mathrm{mmHg}$.

3. Betaxolol 20mg + Ramipril $5 \mathrm{mg}$ BP $150 / 100 \mathrm{mmHg}$.

4. Betaxolol $20 \mathrm{mg}+$ Ramipril $10 \mathrm{mg} \mathrm{BP}$ $140 / 83 \mathrm{mmHg}$.

The first result (1) is in the left lower quadrant (low vagal activity, sympathetic predominance - usually visible in ill persons with inadequate regeneration). Next control result (2) shifts to the left upper quadrant (low vagal activity, low sympathetic activity - usually visible in persons with bad physical and mental condition). Third examination (3) with fall of vagal activity and attenuation of sympatho-vagal balance. The final examination result (4) in the left upper quadrant with shift to right (trend to enhance vagal activity) is in the border of normal range.

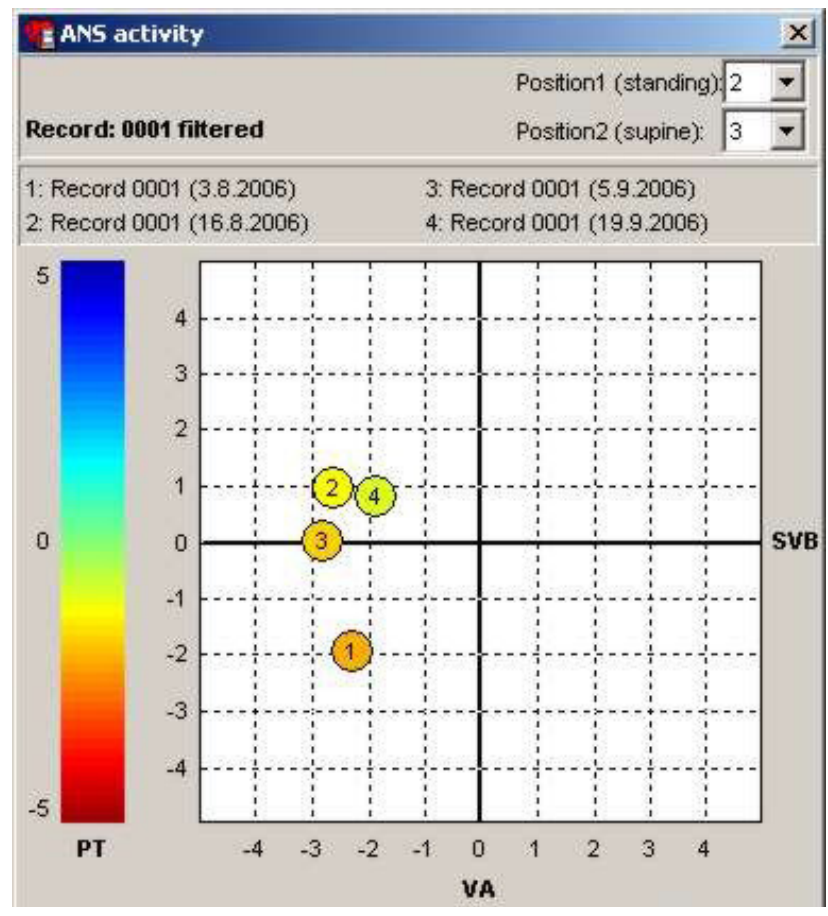

\section{CONCLUSIONS}

HRV is one of reputable prognostic factors of cardiovascular mortality. SA HRV during supine-standingsupine test offers evaluation of sympatho-vagal balance ${ }^{4}$. ANS on the one side participate on etiopathogenesis of hypertension and on the other side is under influence of antihypertensive medicaments. Therefore examination of HRV in hypertensive patients offers next evaluative parameter beside proper blood pressure measurement. This approach allows view of patients and his therapy in more details. Proper examination taking about 30 minutes regards standard conditions, sinus rhythm of patient and high quality recording of RR intervals.

\section{ACKNOWLEDGEMENT}

The author wishes to thank Mr. Leo Rec for his graphic aid.

\section{REFERENCES}

1. Kára T, Souček M. Autonomní nervový systém. In: Souček M, Kára T. et al. Klinická patofyziologie hypertenze, Grada Publishing 2002:37-68

2. Heart rate variability. Standards and measurement, physiological interpretation, and clinical use. The Task Force of the European Society of Cardiology and the North American Society of Pacing and Electrophysiology. Circulation 1996; 93:1043-1065

3. Akselrod S, Gordon D, Madwed JB, et al. Hemodynamic regulation: investigation by spectral analysis. American Journal of Physiology 1985; 249(Part 2):H 867-875

4. Opavský J. Metody vyšetřování autonomního nervového systému a spektrální analýza variability srdeční frekvence $\mathrm{v}$ klinické praxi. In: Variabilita srdeční frekvence a její hodnocení v biomedicínckých oborech - od teorie ke klinické praxi IV. odborný seminár s mezinárodní účastí. Sborník článků a abstrakt. Jiř́i Salinger (editor) Univerzita Palackého v Olomouci Fakulta tělesné kultury Olomouc 2004: 81-85

5. Stejskal P. Využití nové metodiky hodnocení SA HRV pomocí komplexních indexů v klinické a sportovní praxi. In: Variabilita srdeční frekvence a její hodnocení v biomedicínckých oborech - od teorie ke klinické praxi IV. odborný seminář s mezinárodní účastí. Sborník článků a abstrakt. Jiří Salinger (editor) Univerzita Palackého v Olomouci Fakulta tělesné kultury Olomouc 2004: 105-116

6. Opavský J. Metodika spektrální analýzy variability srdeční frekvence v krátkodobých záznamech. In: Opavský J. Autonomní nervový systém a diabetická autonomní neuropatie. Klinické aspekty a diagnostika:164-169

7. Salinger J, Opavský J, Bůla J, et al. Programové vybavení měřicího systému, typ TF-2, určené pro spektrální analýzu intervalů v kardiologii. Lékař a technika, 1994, 25: 58-62

8. Salinger J, Pumprla J, Vychodil R, Stejskal P, Opavský J, Novotný $\mathrm{J}$, et al. Microcomputer system for telemetric assessment of short term heart rate variability in time and frequency domain, Type Varia Cardio TF 4. In: Murray A, Swiriyn S. Computers in Cardiology, Los Alamitos: The Institute of Electrical and Electronics Engineers, Computer Society Press; 1999. p. 599-602.

9. YamamotoY., Hughson RL. Coarse-graining spectral analysis: new method for studying heart rate variability. Journal of Applied Physiology. 1991, 71: 1143-1150

10. Šlachta R. Stejskal P, Stejskal D, Bure J, Elfmark M, Kalina M, et. al. Application of age-standardised parameters in the evaluation of SA HRV in clinical practice. Gymnica 2000; 30 (1): 41-48

11. Salinger J, Stejskal P, Opavský J, Gwozdziewicz M, Gwozdziewiczová S, Novotný J, Elfmark M, Bula J. System type VarCor PF for non-invasive diagnostics of the heart rate variability and of the respiratory rate. In: Variabilita srdeční frekvence a její hodnocení $\mathrm{v}$ biomedicínckých oborech - od teorie ke klinické praxi IV. odborný seminář s mezinárodní účastí. Sborník článků a abstrakt. Jiří Salinger (editor) Univerzita Palackého v Olomouci Fakulta tělesné kultury Olomouc 2004: 96-104 